\title{
Edge Point Grouping for Line Detection
}

SUMMARY This paper proposes a method of accurately detecting the boundary of narrow stripes, such as lane markings, by employing gradient cues of edge points. Using gradient direction cues, the edge points at the two sides of the boundary of stripes are classified into two groups before the Hough transform is applied to extract the boundary lines. The experiments show that the proposed method improves significantly the performance in terms of the accuracy of boundary detection of narrow stripes over the conventional approaches without edge point grouping.

key words: driving assistance, lane marking detection, Hough transform, and gradient direction

\section{Introduction}

Lane marking detection is a basic task for driving assistance. Since lane markings are often detected from their two side boundaries, the accuracy on the boundary detection of lane markings determines the performance of lane marking detection directly.

For the boundary detection of straight lane markings, the Hough transform is a popular method and is widely used in many studies.

\subsection{Conventional Approach without Edge Point Grouping}

The conventional approach of the detection of straight lines is shown as the process (1) in Fig. 1. Figure 1 (a) shows a synthesized image, in which there are three horizontal stripes with different width. The edge image obtained by the Canny operator is shown in Fig. 1 (b).

The boundary lines of the stripes are detected using the SHT (Standard Hough Transform) [1], [2], and are indicated in red color, as shown in Fig. 2 (a). While the lines of the boundary of the stripes with relatively wide width are extracted correctly, multiples lines are extracted from the boundary of the narrowest one near the top; some of them cross the two sides of the boundary of the narrow stripe. In this paper, this case is called the narrow stripe line detection problem.

What causes the narrow stripe line detection problem above? Note that in the SHT, only the position cues, $\left(x_{i}, y_{i}\right)$, of edge points are used for the vote in the parameter space $(\rho, \theta)$. For the lines nearing each other, such as the two sides of the boundary of a narrow stripe, the corresponding two

Manuscript received September 29, 2011.

Manuscript revised January 8, 2012.

†The author is with the Graduate School of Engineering, Tottori University, Tottori-shi, 680-8552 Japan.

a)E-mail: li@ele.tottori-u.ac.jp

DOI: 10.1587/transinf.E95.D.1713 peaks of the maximum of the accumulator array become very close, and this makes it difficult to distinguish the two peaks in the parameter space. Figure 3 shows the vote space of the edge points of Fig. 1 (b). While the peaks corresponding to the two lines of the boundary of the other two horizontal stripes, enclosed by green circles in Fig. 3, are obviously separated from each other, the peaks corresponding to the narrowest horizontal stripe, enclosed in red circle, approach each other, so that we fail to identify the two peaks correctly in the vote space.

\subsection{Related Research}

The Hough transform is a very popular approach for line extraction. Recently, to robustly detect lines in noisy environments, a method of adding prior modeling on the Hough parameters has been proposed [3]. For fast line segment, the meanshift clustering approach in Hough domain is proposed [4]. A statistical kernel modeling of the Hough transform is proposed so that the resulting estimate of parameters is continuous and includes as much information as available [5]. In some of these approaches [5], [6], gradient cues are used to improve the accuracy of the parameter $(\rho, \theta)$. However, since the edge points at the two sides of the boundary are not grouped beforehand, the narrow stripe line detection problem still remains in the above approaches.

\subsection{Proposed Method}

If we can classify the edge points of the two sides of the boundary before voting, the lines corresponding to the boundary can be detected more accurately.

In this paper we propose a method of employing the gradient cues of edge points to detect lines more accurately by grouping the edge points before applying the Hough transform. The proposed method can cope with the above problem effectively.

Figure 4 shows a sketch of an image that includes a bar. The edge points at the two sides of the boundary are indicated by $\left(x_{k}^{p}, y_{k}^{p}\right)$ and $\left(x_{j}^{n}, y_{j}^{n}\right)$. Their gradient direction is indicated by red and green arrows, respectively. Note that while the angle of the gradient direction of $\left(x_{k}^{p}, y_{k}^{p}\right)$ is ideally equal to parameter $\theta$ of the Hough transform, the gradient direction of $\left(x_{j}^{n}, y_{j}^{n}\right)$ is opposite. If we can classify the edge points of the two sides of the boundary of the bar based on their gradient direction into two different groups and carry out the vote separately, the narrow stripe line detection prob- 
(b) Edge Image without grouping

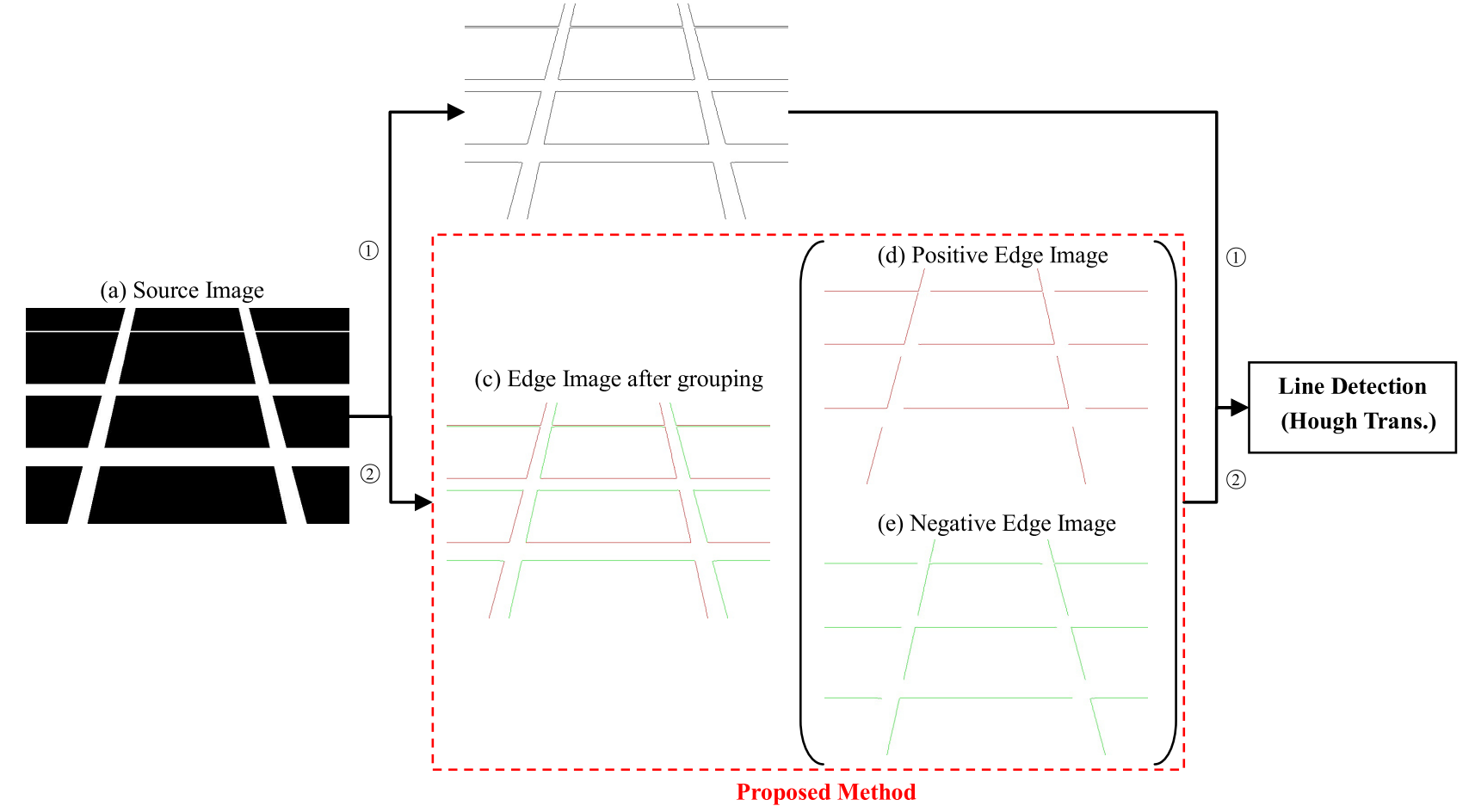

Fig. 1 The sketch for the process of line detection. The conventional approach without edge point grouping is indicated by (1); the process of the proposed method with edge point grouping is indicated by (2).

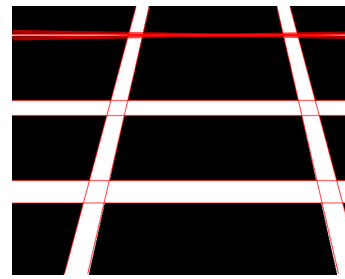

(a)
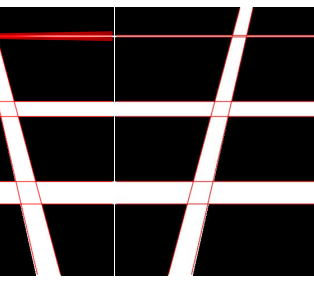

(b)

Fig. 2 (a) Lines (indicated in red) detected by the conventional approach without edge point grouping. (b) Lines detected by the proposed method with edge point grouping.

lem can be eliminated.

The process of the proposed method is shown as (2) in Fig. 1. Figure 1 (c) shows the edge image after grouping, where the upper edge points, as shown in Fig. 1(d), and lower edge points, as shown in Fig. 1 (d), are indicated by red and green colors, respectively. The lines detected from Fig. 1 (c) are shown in Fig. 2(b); all the lines are detected correctly. Note that for both processes (1) and (2) the same line detection algorithm SHT is applied with the same parameters, such as the size of Hough space and so on. The differences in Fig. 2 (a) and (b) stem from whether the edge points are grouped or not before the SHT is applied.

The remainder of this paper is organized as follows. We explain the proposed method in Sect. 2. After presenting the experimental results in Sect. 3, we give our conclusions in Sect. 4.

\section{Line Detection by Edge Point Grouping}

\subsection{Grouping Edge Points by Gradient Direction}

Given an edge point $\left(x_{i}, y_{i}\right)$ and its corresponding Hough transform parameters $(\hat{\rho}, \hat{\theta})$, in terms of

$$
\rho=x \cos \theta+y \sin \theta
$$

we have

$$
\hat{\rho}=A \sin (\hat{\theta}+\gamma),
$$

where $A=\sqrt{x_{i}^{2}+y_{i}^{2}}$ and $\gamma=\arcsin \left(\frac{x_{i}}{\sqrt{x_{i}^{2}+y_{i}^{2}}}\right)$. Because $\sin (\hat{\theta}+\gamma) \geq 0$,

$$
0 \leq \hat{\theta}+\gamma \leq \pi
$$

Let $\beta=\arctan \left(\frac{y_{i}}{x_{i}}\right)$. Then, $\gamma=\frac{\pi}{2}-\beta$. We have

$$
-\frac{\pi}{2} \leq \hat{\theta}-\beta \leq \frac{\pi}{2} .
$$

Suppose the gradient direction of $\left(x_{j}^{n}, y_{j}^{n}\right)$ and $\left(x_{k}^{p}, y_{k}^{p}\right)$ in Fig. 3 are $\alpha_{j}^{n}$ and $\alpha_{k}^{p}$, respectively. Because $\alpha_{k}^{p}=\hat{\theta}$ for $\left(x_{j}^{n}, y_{j}^{n}\right)$, theoretically, Eq. (5) can be rewritten as

$$
-\frac{\pi}{2} \leq \alpha_{k}^{p}-\beta \leq \frac{\pi}{2}
$$




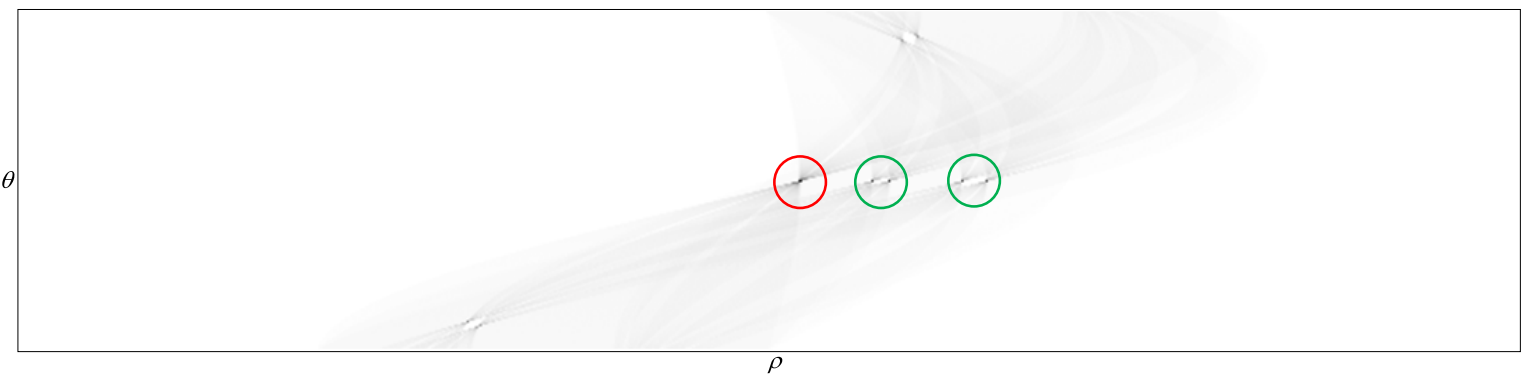

Fig. 3 Accumulator array of the voting. The peaks corresponding to the narrowest horizontal stripe are enclosed by a red circle while that of other two horizontal stripes are enclosed by green circle.

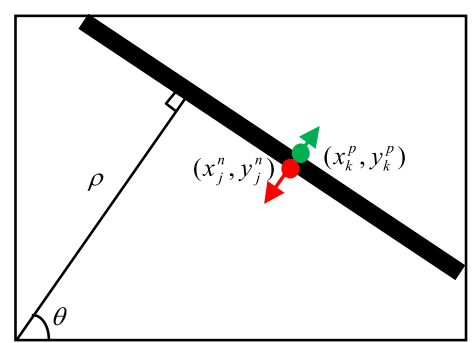

Fig. 4 Line detection for a bar.

Thus,

$$
\cos \left(\alpha_{k}^{p}-\beta\right) \geq 0 \text {. }
$$

On the other hand, for $\left(x_{j}^{n}, y_{j}^{n}\right), \alpha_{j}^{n}=\hat{\theta}+\pi$. Then, in terms of Eq. (5) we have

$$
\cos \left(\alpha_{j}^{n}-\beta\right) \leq 0 .
$$

Therefore, the edge points at the two sides of the boundary of a stripe can be grouped in terms of (6) and (7). If the Hough transform is applied to the edge points of the two groups, respectively, the narrow stripe line detection problem described in Sect. 1 can be removed definitely.

\subsection{Detailed Algorithm}

Here, we give the detailed algorithm of the proposed algorithm as follows.

- Given an input image $I(x, y)$, compute the gradient direction $\left\{\alpha_{i}\right\}$ of the edge points $S=\left\{\left(x_{i}, y_{i}\right)\right\}$.

- For edge point $\left(x_{i}, y_{i}\right)$, compute its polar angle $\beta_{i}$.

- If $\cos \left(\alpha_{i}-\beta_{i}\right) \geq 0$, the edge point $\left(x_{i}, y_{i}\right)$ is assigned to group $S_{P}=\left\{\left(x_{k}^{p}, y_{k}^{p}\right)\right\}$. Otherwise, the edge point $\left(x_{i}, y_{i}\right)$ is assigned to group $S_{n}=\left\{\left(x_{j}^{n}, y_{j}^{n}\right)\right\}$.

- Perform voting on each group separately $S_{n}=$ $\left\{\left(x_{j}^{n}, y_{j}^{n}\right)\right\}$ and $S_{P}=\left\{\left(x_{k}^{p}, y_{k}^{p}\right)\right\}$, respectively.

Concretely, two accumulator arrays $A_{n}(\rho, \theta)$ and $A_{p}(\rho, \theta)$ are created for $S_{n}$ and $S_{p}$, respectively. The vote for the two accumulator arrays is carried out as follows.

$$
\begin{array}{lll}
A_{p}(\rho, \theta)=A_{p}(\rho, \theta)+1 & \text { if } & \cos \left(\alpha_{i}-\beta_{i}\right) \geq 0 \\
& \& & x_{i} \cos \theta+y_{i} \sin \theta=0 ; \\
A_{n}(\rho, \theta)=A_{n}(\rho, \theta)+1 & \text { if } & \cos \left(\alpha_{i}-\beta_{i}\right)<0 \\
& \& & x_{i} \cos \theta+y_{i} \sin \theta=0 .
\end{array}
$$

\section{Experimental Result}

As shown in Fig. 1, the proposed method groups the edge points in term of their gradient direction; it can be used as a pre-processing for the conventional Hough-transform-based line detection algorithms [1]-[6]. Here, the sample program of the SHT included in the well-known OpenCV [7] is used for this test.

A source image of road scene including narrow lane markings is shown in Fig. 5 (a); the image size is $400 \times 300$ pixels. The edge image after grouping by the proposed method is shown in Fig. 5 (b), where the edge points belonging to different groups are indicated by different colors. The lines detected using all the edge points without grouping (the conventional method) are shown in Fig. 5(c); the lines detected using the grouped edge points (the proposed method) are shown in Fig. 5 (d). For the SHT, the resolution of $\rho$ is 1 pixel; the resolution of $\theta$ is 1 degree. The seven greatest peaks in the parameter space are extracted, which correspond to the seven lines.

In Fig. 5 (c), four lines, No.2, No.3, No.4 and No.5, are extracted from the narrow lane marking on the left; among them No.2 and No.3 are extracted from the different groups of the edge points. In contrast, two lines corresponding to the boundary of the narrow lane marking are extracted correctly as No.2 and No.3 in Fig. 5 (d) by using the grouped edge points; additionally, the boundary lines, No.4 and No.5, of the central broken lane marking are extracted as one of the seven greatest peaks from the parameter space.

To evaluate the experimental results quantitatively, we compute the vanishing point of the extracted lines which are parallel to each other at space, and use the residual error of the estimate to measure the accuracy of the extracted lines. For line $l_{i}$,

$$
a_{i} x+b_{i} y+c_{i}=0 \quad(i=1, \cdots, N)
$$

Where $N$ is the number of the parallel lines. The vanishing point $\left(x_{v}, y_{v}\right)$ is computed from the following equation by the least square method.

$$
\sum_{i=1}^{N}\left(a_{i} x_{v}+b_{i} y_{v}+c_{i}\right)^{2}=0
$$




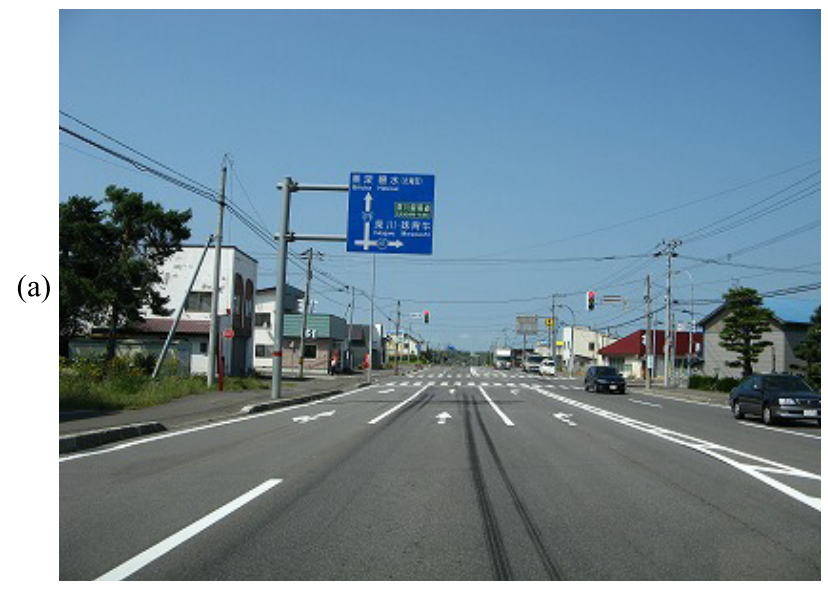

(b)
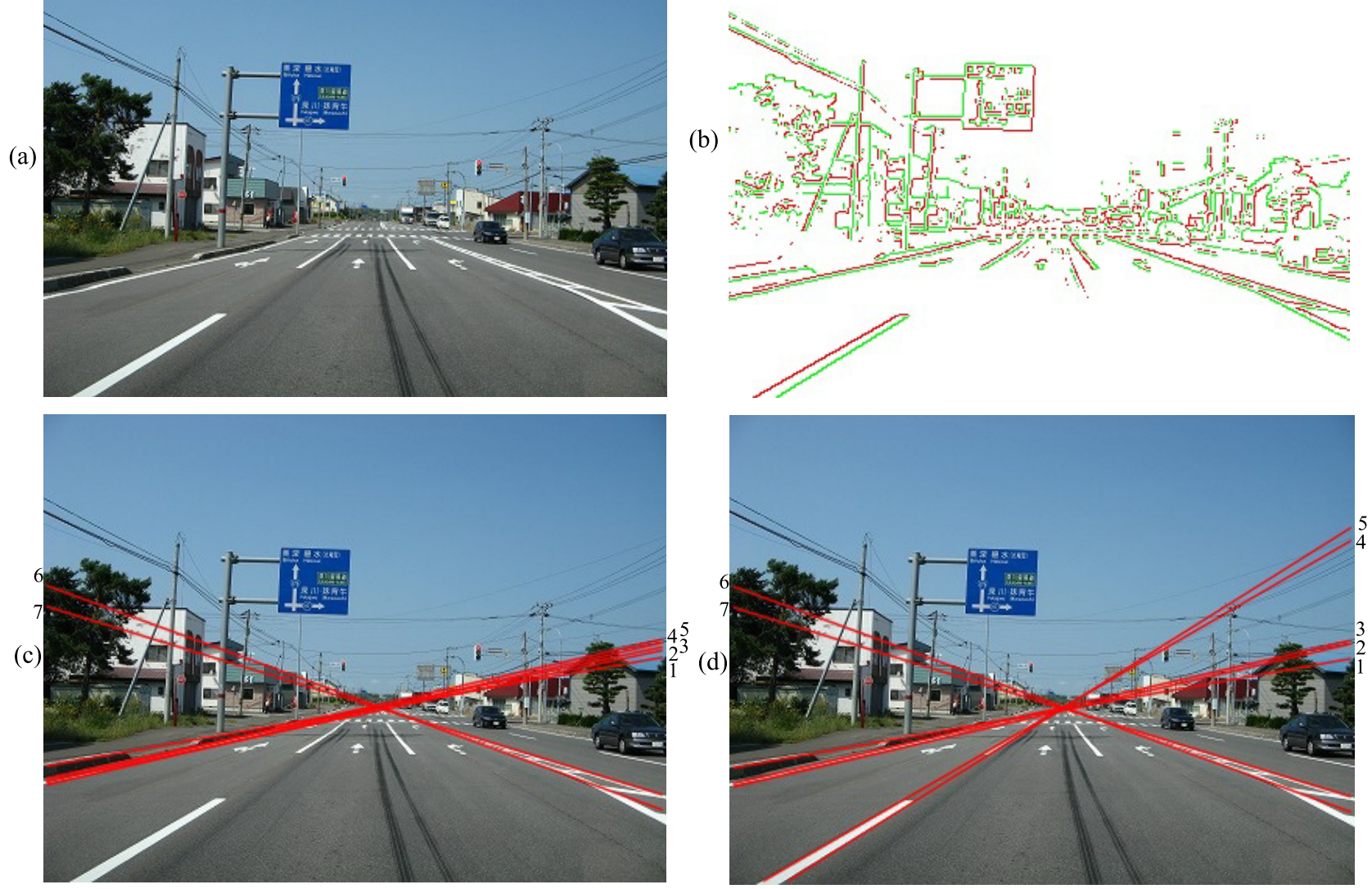

Fig.5 (a) Source image. (b) Grouped edge points. (c) Extracted lines without edge point grouping.

(d) Extracted lines with edge point grouping.

The residual error normalized by the line number is computed as

$$
E=\frac{1}{N} \sum_{i=1}^{N}\left(a_{i} \hat{x}_{v}+b_{i} \hat{y}_{v}+c_{i}\right)^{2} .
$$

While the residual error computed from the lines of Fig. 5 (c) is 1.223550 pixels, the residual error computed from the lines of Fig. 5 (d) decreases to 0.579347 pixels. More experimental results can be found in [8].

\section{Conclusions}

Edge point grouping followed by the Hough transform is a very simple, but very practical technique. The proposed method can be used to the conventional Hough-transformbased line detection methods as a pre-processing. As one of the applications, the proposed method can be used to detect narrow lane markings for driving assistance.

\section{References}

[1] P. Hough, "Methods of means for recognizing complex patterns," US Patent 3069 654, 1962.

[2] R.O. Duda and P.E. Hart, "Use of the Hough transformation to detect lines and curves in pictures," Commun. ACM, vol.15, pp.11-15, 1972.

[3] N. Aggarwal and W.C. Karl, "Line detection in image through regularized Hough transform," IEEE Trans. Image Process., vol.15 no.3, pp.582-591, 2006.

[4] A. Bandera, J. Perez-Lerenzo, and F. Sandoval, "Meanshift based clustering of Hough domain for fast line segment detection," Pattern Recognit. Lett., vol.27, pp.578-586, 2006.

[5] R. Dahyot, "Statistical Hough transform," IEEE Trans. Pattern Anal. Mach. Intell., vol.31, no.8, pp.1502-1509, 2009.

[6] Q. Ji and R.M. Haralick, "Error propagation for the Hough transform," Pattern Recognit. Lett., vol.22, pp.813-823, 2001.

[7] http://sourceforge.net/projects/opencvlibrary/

[8] http://www.skybird.ele.tottori-u.ac.jp/edgeGroupingData.pdf 